\title{
Influence of Stresses on Precipitation
}

\author{
G. Sauthoff \\ Max-Planck-Institut für Eisenforschung GmbH, Postfach 140444, 40074 Düsseldorf, Germany
}

\begin{abstract}
Precipitation, which usually occurs by nucleation, growth and coarsening, may be affected by elastic stresses which influence the thermodynamics of the system and thereby the driving force for precipitation. Such stresses may be internal, e.g. coherency stresses, or external, i.e. externally applied stresses. The consequences for nucleation, growth and coarsening are discussed in detail with special emphasis on the kinetics of precipitation. The possible effects are exemplified by various cases of the orienting of precipitate particles by external stress. The effect of elastic stress on phase solubility may not only affect precipitation by nucleation and growth, but also spinodal decomposition which is briefly discussed. Larger elastic stresses, which surpass the yield stress, result in plastic deformation with production and movement of dislocations. Dislocations may affect precipitation processes by their stress fields, by being incorporated into interfaces to lower the interface energy and by providing easy diffusion paths. Again the effects are discussed with respect to nucleation, growth and coarsening. The effects are made use of for alloy processing by thermomechanical treatments, and furthermore they may lead to structure changes - with corresponding changes in mechanical behaviour - during high-temperature service with creep.
\end{abstract}

\section{INTRODUCTION}

In many materials precipitated particles are used for controlling the physical properties and in particular the mechanical behaviour. The fundamentals of the various precipitation processes have been studied since long and are well understood as is documented in respective reviews and monographs - see e.g. [1] where further references were given. Precipitation is usually regarded as a sequence of three stages, i.e. nucleation, growth and coarsening of particles. For all three processes simple theoretical descriptions are available which regard the precipitated particles - even the very small nuclei - as bulk phases to which the thermodynamics of multiphase systems - see e.g. [2] - can be applied. The kinetics of these processes, i.e. the rates of nucleation, growth and coarsening, are controlled by the driving force for precipitation, which is a function of the free enthalpies - or Gibbs energies - of the phases involved, and the atom mobilities, which are described by the respective diffusion coefficients.

The formation of particles may lead to internal elastic stresses, which are known as coherency stresses. Such stresses contribute to the particle energy and thus change the equilibrium conditions and correspondingly the driving force for the various precipitation processes. Externally applied stresses, which produce elastic strains in the crystal, may directly affect the equilibrium conditions between the phases involved and thus again change the driving force. Stresses that surpass the yield stress of the material cause plastic deformation with formation and movement of dislocations. Dislocations are linear defects, which may interact with particles because of their line energy or their cylindrical stress fields, and furthermore they may be paths of easy diffusion.

The present report is to give an overview on the various effects. The physical understanding of these effects is not only of academic interest, but also of practical importance with respect to materials processing - in particular thermomechanical treatments - on the one hand and to materials service behaviour - in particular long-time service under creep conditions - on the other hand. 


\section{PRECIPITATION BY NUCLEATION, GROWTH AND COARSENING}

\subsection{Nucleation}

A phase $\beta$ precipitates from a solid solution $\alpha$, which is then the matrix, if the solubility of $\beta$ in $\alpha$ is decreased by lowering the temperature to such an extent that the matrix is supersaturated with respect to $\beta$ - see [1]. The driving force for any reactions between two phases is given by the difference in Gibbs energy of the respective phases. In the case of small particles and nuclei the interface energy is not negligible and has to be considered. The resulting energy difference - or transformation energy $\tilde{g}$ (per alloy atom) - can be determined in simple approximation as shown in Fig. 1. In many practical cases the matrix can be regarded as dilute solid solution and the precipitate phase as compound with a narrow composition range. Then $\tilde{g}$ is given approximately by

$$
\widetilde{g}=k T x_{c}^{\beta} \ln \frac{x^{\alpha}}{x_{e}^{\alpha}}
$$

Eq. 1

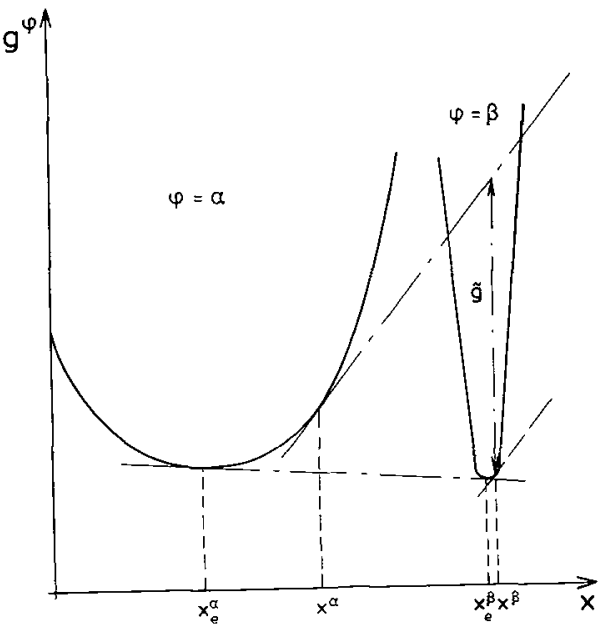

Figure 1: The Gibbs energies $g$ of phases $\varphi=\alpha, \beta$ as a function of alloy content $x$ at constant temperature with transtormation energy $\widetilde{g}$ (schematic) [1]. where the compositions with index e refer to equilibrium as given in a phase diagram, the composition ratio $\frac{x^{\alpha}}{x_{e}^{\alpha}}$ is the supersaturation, and kT has its usual meaning.

The particle formation energy - or nucleation energy -, which is composed of the volume energy and the surface energy of the particle, reaches a maximum, which is known as the critical nucleation energy $A^{*}$, for a critical size, above which the particle grows with decreasing energy. For spherical particles $A^{*}$ is calculated as

$$
A^{*}=\frac{16 \pi}{3} \frac{\gamma^{3}}{\left(\tilde{g} / v^{\beta}\right)^{2}}
$$

Eq. 2

where $\gamma=$ specific particle surface energy and $v^{\beta}$ $=$ particle atomic volume. The primary importance of the surface energy is clearly visible.

The nucleation rate is controlled by the critical nucleation energy which is the energy barrier for nucleation. Accordingly the relation between the nucleation rate $J$ and $A^{*}$ is given by an Arrhenius-type equation:

$$
J=\kappa^{\prime} \beta^{*} N_{0} \exp \left(-A^{*} / k T\right)
$$

with $N_{o}=$ nucleation site concentration, $\beta^{*}=$ frequency factor proportional to the diffusion coefficient, and $\kappa^{*}=$ factor of proportionality -

for details and references see [1]. It is noted that this equation was derived for stationary nucleation, i.e. constant supersaturation with constant nucleation rate. Nevertheless this simple equation is useful for describing the nucleation process as a function of temperature, alloy composition and external forces at least in a semi-quantitative way in spite of the various approximations of the used model of classical nucleation.

Finally it is noted that besides nucleation the alloy may decompose by spinodal decomposition, i.e. particles form by the continuous growth of small concentration fluctuations without any thermal acti- 
vation [3]. This spinodal decomposition is expected only for alloys with compositions between the socalled spinodal lines within the miscibility gap in the respective phase diagram which is not the case for most practical alloys.

\subsection{Growth}

After nucleation particles grow by the consumption of solute atoms, which diffuse from the supersaturated $\alpha$ matrix to the growing $\beta$ particle. The reactions at the interface are usually much faster than the diffusion to the particle, i.e. the diffusion to the particle is the rate controlling process. Furthermore local equilibrium is usually assumed for the growing particle which is described by using the thermodynamics of heterogeneous equilibria as in the case of the critical nucleus. In view of the relation between critical nucleus radius and transformation energy, the matrix concentration in equilibrium with the growing particle - i.e. the particle solubility in the matrix - is given by

$$
c^{\alpha \beta}(r)=c_{e}^{\alpha} \exp \left(2 \gamma / c_{e}^{\beta} k T r\right) \text {, }
$$

Eq. $4 \mathrm{a}$

which is the well-known Gibbs-Thomson equation for spherical particles with radius $r$. Correspondingly the actual matrix composition $c^{\alpha}$ is related to the critical particle radius $r^{*}$ by

$$
c^{\alpha}(t)=c_{e}^{\alpha} \exp \left(2 \gamma / c_{e}^{\beta} k T r^{*}\right)
$$

Eq. 4b

- see [1].

The mass balance gives a differential equation for particle growth which can be integrated in a simple way if on the one hand matrix depletion by the growing particle is neglected, which may be adequate small particles, and if on the other hand $c^{\alpha \beta}(r)$ may be approximated by $c_{e}^{\alpha}$, which may be adequate for not too small particles. This leads to the familiar parabolic growth law

$$
r^{2}-r_{0}^{2}=\frac{c_{0}-c_{e}^{\alpha}}{c_{e}^{\beta}-c_{e}^{\alpha}} 2 D\left(t-t_{0}\right),
$$

where $D$ is the effective diffusion coefficient in the matrix, $t$ is the time, and the index 0 indicates the initial state - see [1].

For continued growth the matrix depletion - i.e. the decrease of supersaturation by the growing particles - has to be considered. A simple treatment for a constant number of particles with equal size leads to the familiar Johnson-Mehl or Avrami equation for the precipitated volume fraction

$$
W(t)=1-\exp \left(-(t / \tau)^{n}\right)
$$

with an exponent $n=3 / 2$ for the parabolic growth of spherical particles and a time constant $\tau$ which is a function of the initial supersaturation and is inversely proportional to the effective diffusion coefficient. This equation is rather approximate in view of the various simplifying assumption and is useful as a phenomenological relation with fit parameter $n$ and $\tau$.

\subsection{Coarsening}

During nucleation the particles are not formed simultaneously. Thus later formed particles are smaller and have a higher solubility according to Eq. 4a - than earlier formed ones. Then the larger particles represent deeper sinks for solute and grow faster than the smaller ones as is illustrated by Fig. 2a. Simultaneously the matrix composition decreases because of the general particle growth, and likewise the corresponding critical radius increases. The different growth rates of the particles with different sizes then lead to the situation of Fig. $4 \mathrm{~b}$ where the particles with radii smaller than the critical radius have higher solubilities than the matrix composition and thus dissolve whereas only the larger particles with radii larger than the critical radius show continued growth. This process of particle coarsening with a continuously decreasing number of particles because of the dissolution of the smallest ones and increasing average particle size because of the growth of the remaining particles is known as Ostwald ripening, and the driving force for this is the reduction of interface energy. 
The coarsening process is described quantitatively by the theory of Ostwald ripening, which was developed independently by Wagner and by Lifshitz and Slezov and which supposes a negligible supersaturation $c^{\alpha}(t) / c_{e}^{\alpha} \approx 1$ - as is found during the later stages of precipitation - and a small constant volume fraction of precipitate - see [1]. The main results of the theory are the $t^{1 / 3}$ law for the diffusion-controlled average growth of spherical particles:

$$
\bar{r}^{3}-\bar{r}_{0}^{3}=\frac{8}{9} \frac{D \gamma c_{e}^{a}}{c_{e}^{\beta 2} k T}\left(t-t_{0}\right)
$$

and the corresponding law for the particle number:

for negligible $r_{o}$ and $t_{0}$.

$$
N(t)=\text { const. } / t
$$

Eq. 8

\subsection{Precipitate Evolution}

The three classical precipitation stages nucleation, growth and coarsening occur sequentially and par-

a)

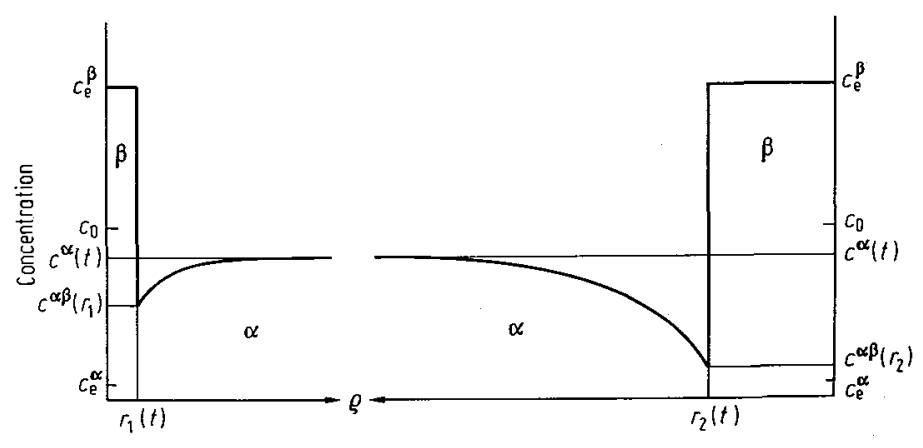

b)

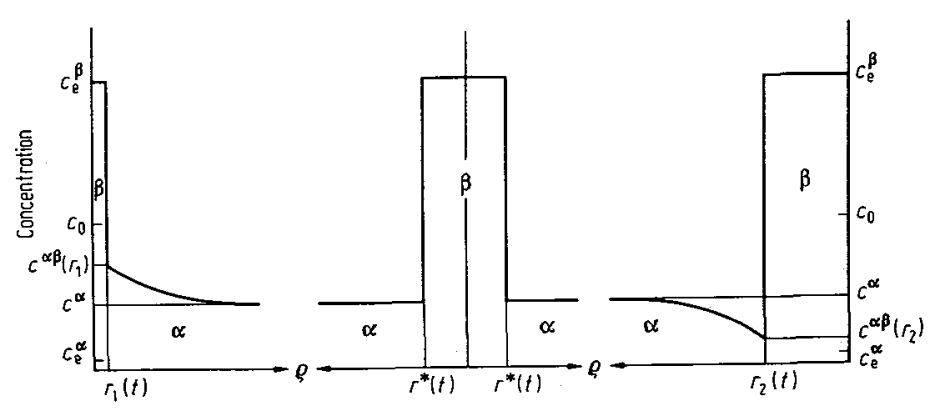

Figure 2: Concentration profile at growing (a) and growing and dissolving particles (b) (schematic) [1]. tially in parallel since the nucleation and growth processes are independent of each other and control the overall precipitation kinetics. The initial undercooling establishes a supersaturation which initiates nucleation with increasing particle number. Then the continuing nucleation and the growth of the already formed particles decrease the supersaturation until nucleation stops. The further growth of the formed particles decreases the supersaturation to such an extent that the smallest particles, which have been nucleated last, become subcritical and start to dissolve. This initiates Ostwald ripening with decreasing particle number. Thus the three precipitation processes principally occur in parallel at the beginning of precipitation and cease one after the other with decreasing supersaturation until 
there is only particle coarsening - see [1]. The decreasing supersaturation corresponds to a decreasing driving force, i.e. the later precipitation stages usually occur with lower reaction rates then the earlier stages. In particular, nucleation is fast compared with growth and coarsening, and growth and coarsening slows down with increasing particle size.

The evolving precipitate distribution is determined by the ratio of the rates of nucleation, growth and coarsening. With lower initial supersaturation or inhibited nucleation only few particles are formed giving rise to a rather coarse particle distribution. With high initial supersaturations many particles are nucleated with high rates which leads to a fine particle distribution after a short growth stage. The further particle coarsening can be accelerated by increasing the coarsening temperature which increases the critical particle size abruptly with the corresponding dissolution of all particles with sizes below this critical size and additionally increases the coarsening rate. In complex alloy systems - in particular steels - various phases with slightly different solubilities may precipitate simultaneously. Then a more general ripening of particles occurs not only with respect to particle size with elimination of the smaller particles, but also with respect to phase stability with elimination of the less stable phase with higher solubility, which may be regarded as generalized Ostwald ripening.

\section{EFFECTS OF ELASTIC STRAIN}

\subsection{Nucleation}

As already mentioned at the beginning, both internal and external stresses may be present during precipitation. In particular, there may be a misfit between the matrix and the particle to be formed. The misfit is accommodated elastically as long as the misfit is not too large, i.e. the resulting elastic stresses do not surpass the yield stress. Such coherency stresses result in extra energy contributions for particle formation and reduce the transformation energy which increases the activation energy for nucleation and the particle solubility for growth and coarsening. This elastic energy depends only on the particle volume for isotropic crystals whereas it depends on the particle shape, too, for anisotropic crystals thus favouring plates for misfits only in one direction and needles for misfits only in two directions. It is noted that the stress fields of the particles may interact with each other, and the resulting interaction energies also contribute to the total particle energy which again reduces the transformation energy. A more detailed discussion of the various effects with simple energy estimates is found in $[1,4,5]$. In a more general way coherency stresses may even affect whether precipitation or ordering or a displacive transformation will occur [6].

External stresses strain the material elastically as long as the stresses do not surpass the yield stress. The resulting elastic strains may be different for matrix and particles if the particles are surrounded by coherency stress fields or show different elastic moduli. In any case the application of the external stress with elastic strain leads to additional interaction energies which contribute to the transformation energy and thereby to the critical nucleation energy as well as to the particle solubility, i.e. all three precipitation stages may be affected depending on the magnitude of the interaction energies - see [5].

As a rule, the elastic interaction energies are small compared with the total driving force of precipitation, i.e. the initial transformation energy at the onset of nucleation. Nevertheless even small changes in the critical nucleation energy (Eq. 2) can affect the nucleation rate significantly because of the exponential dependence of the rate on the activation energy (Eq. 3). This is illustrated by the case of the precipitation of $\alpha$ “- $\mathrm{Fe}_{i \sigma^{\circ}} \mathrm{N}_{2}$ plate-like particles in $\mathrm{Fe}-\mathrm{N}$ alloys [7], which was analysed in detail [8].

The analysis of the $\alpha .-\mathrm{Fe}_{16} \mathrm{~N}_{2}$ precipitation process in $\mathrm{Fe}-\mathrm{N}$ alloys relied on experimentally determined particle numbers as a function of initial supersaturation. From these data the variation of nucleation rates were estimated, and the respective transformation energies were deduced by means of Eq. 1. Then the plot of the nucleation rates as a function of the transformation energies indicated an interface energy of $17 \mathrm{~mJ} / \mathrm{m}^{2}$ which is a reasonable value for coherent particles. With these data critical nucleation energies of about $200 \mathrm{MJ} / \mathrm{m}^{3}$ were obtained whereas the elastic interaction energy was only about $10 \mathrm{MJ} / \mathrm{m}^{3}$ for the platelets perpendicular to the external stress for a tetragonal misfit of $10 \%$ in plate 
normal direction and a stress of about $100 \mathrm{MPa}$. This $5 \%$ advantage of the ,right" particles perpendicular to the external stress over the „wrong“ particles leads to preferential nucleation of the "right" particles, i.e. the nucleation rate of the "right" particles increased by a factor of about 45 . Then a rather complete particle orienting would be expected at the end of nucleation with about $95 \%$,right" particles and still more up to $100 \%$ for decreasing initial transformation energy, i.e. initial supersaturation. Indeed a rather complete particle orienting was observed experimentally which confirms the applicability of the applied simple estimates. However, a complete orienting was obtained only for the highest supersaturation after longer ageing. Obviously the observed complete orienting had not been produced by nucleation exclusively, and the later precipitation stages were needed for completing the orienting process which will be addressed in the next section.

\subsection{Growth and Coarsening}

During growth and coarsening the matrix supersaturation decreases continuously and likewise the transformation energy to approach zero asymptotically after long times. If particles are sources of coherency stresses, which surpass the order of the yield stress, the particles become incoherent, i.e. the stresses are relaxed by misfit dislocations in the interface, which occurs at a critical particle size after nucleation. This reduces the particle volume energy, i.e. it increases the transformation energy and thereby reduces the particle solubility on the one hand, and on the other hand it increases the interface energy. The former increases the growth rate and decreases the coarsening rate (Eqs. 5 and 7) whereas the latter increases the driving force for coarsening and thereby the coarsening rate (Eq. 7). The effects of stress on coarsening have recently been studied theoretically by computer simulations [ 9 ].

The application of an external elastic stress gives rise to interaction energies of the particles if the particles are surrounded by coherency stress fields or show different elastic moduli as in the case of nucleation. Even small interaction energies can affect the coarsening process decisively since after sufficiently long ageing times such interaction energies may have become larger than the monotonously decreasing transformation energy, and then the interaction energy contribution decides on further growth or dissolution of the individual particle.

This is illustrated by the above discussed case of the $\alpha$ " $-\mathrm{Fe}_{16} \mathrm{~N}_{2}$ plate-like particles in $\mathrm{Fe}-\mathrm{N}$ alloys which was analysed in detail [8]. The nucleation of these particles in the presence of an external elastic stress results in a particle distribution with most particles oriented perpendicular to the stress and few particles with the energetically unfavourable orientation parallel to the stress. Energetically unfavourable means that those particles have a higher solubility than the particles with the favourable orientation and thus dissolve preferentially to the advantage of the favoured particles during coarsening. Thus the Ostwald ripening process here refers to both particle size and particle orientation and results in the continuous elimination of not only the smallest particles, but also of the particles with energetically unfavourable orientation until complete orienting is reaches. For the addressed case this means that additional coarsening with external stress after nucleation is necessary for obtaining the observed complete particle orienting.

The action of this particle orienting process exclusively by Ostwald ripening was demonstrated with the example of the precipitation of Au particles in ferritic Fe-Mo-Au alloys [4,5,10]. As in the case of the $\alpha^{4-}-\mathrm{Fe}_{16} \mathrm{~N}_{2}$ particles in Fe- $\mathrm{N}$ alloys the Au particles are precipitated as platelets with tetragonal distortion which makes the particle orientation perpendicular to an external elastic stress energetically favourable. However, the interaction effects are here smaller and in particular the effect of the external stress on nucleation is only small, i.e. a particle orienting by preferential nucleation of the ,right" particles is not clearly visible after nucleation. Only subsequent coarsening with external stress results in complete orienting of the precipitated particles is illustrated by Figs. 3a and b. A particle coarsening with an increase of the average particle diameter by a factor between 3 and 5 - depending on initial particle size - is sufficient for complete orienting according to the theoretical analysis which was confirmed by the experimental observation. 
a)
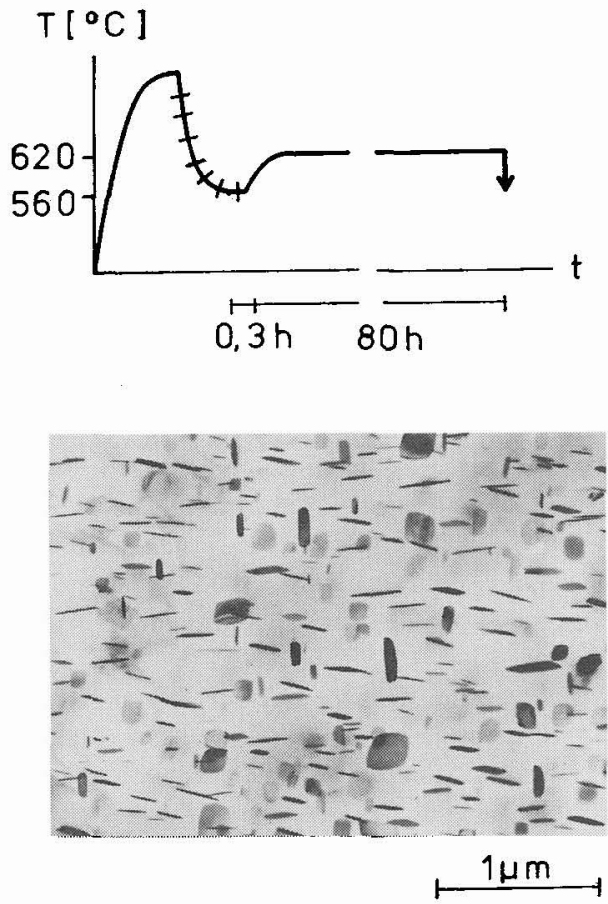

b)
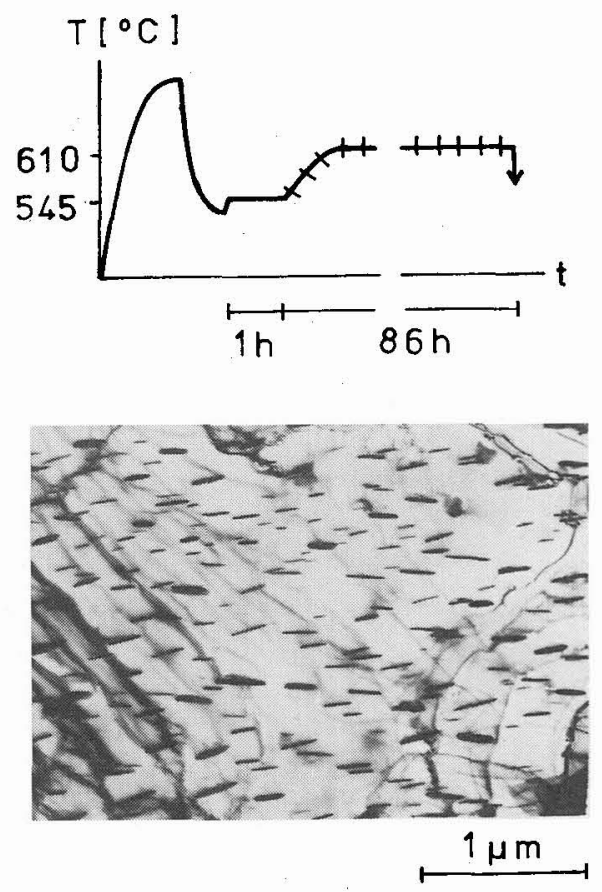

Figure 3: Transmission electron micrographs of precipitated Au particles in a Fe-Mo-Au single crystal with application of an external elastic tensile stress of $25 \mathrm{MPa}$ during nucleation (a) or coarsening (b). The respective temperature vs. time diagrams indicate the used heat treatments with a comparatively low nucleation temperature for establishing a sufficient initial supersaturation for homogeneous nucleation [10].

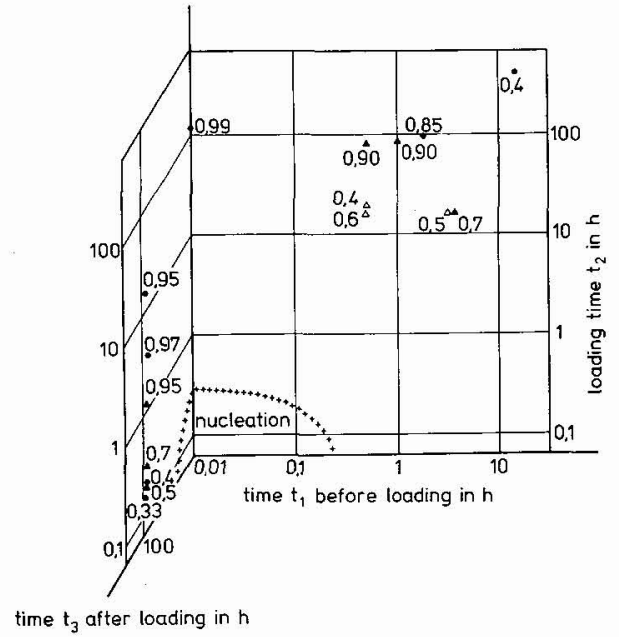

Figure 4: Measured fractions of Au particles perpendicular to the external stress (numbers at the symbols) and end of nucleation stage $(++++++)$ for a Fe-Mo-Au alloy as a function of time $t_{1}$ before stress application, time $t_{2}$ with stress application of $25 \mathrm{MPa}$, and time $t_{3}$ after stress application for various heat treatments see [10].
During coarsening with applied stress the unfavourably oriented "wrong“ particles behave like smaller ,right" particles, and consequently a usually barely visible size difference between „right“ and „wrong“ particles develops during coarsening under stress. However, even a small size difference is sufficient for eliminating the "wrong“ particles by further coarsening without applied stress. This effect was indeed observed experimentally as is visible in Fig. 4.

It is noted that such particle orienting may also be achieved by the application of a magnetic force as was demonstrated previously for the above discussed case of $\alpha^{\prime \prime}-\mathrm{Fe}_{16} \mathrm{~N}_{2}$ precipitation in Fe-N alloys [11]. It could be shown that the observed particle orienting again occurred by selective coarsening and was due to the direct interaction of the external magnetic field with the precipitated particles whereas the spontaneous magnetization was not sufficient for achieving a particle orienting within the magnetic domains $[12,13]$. 


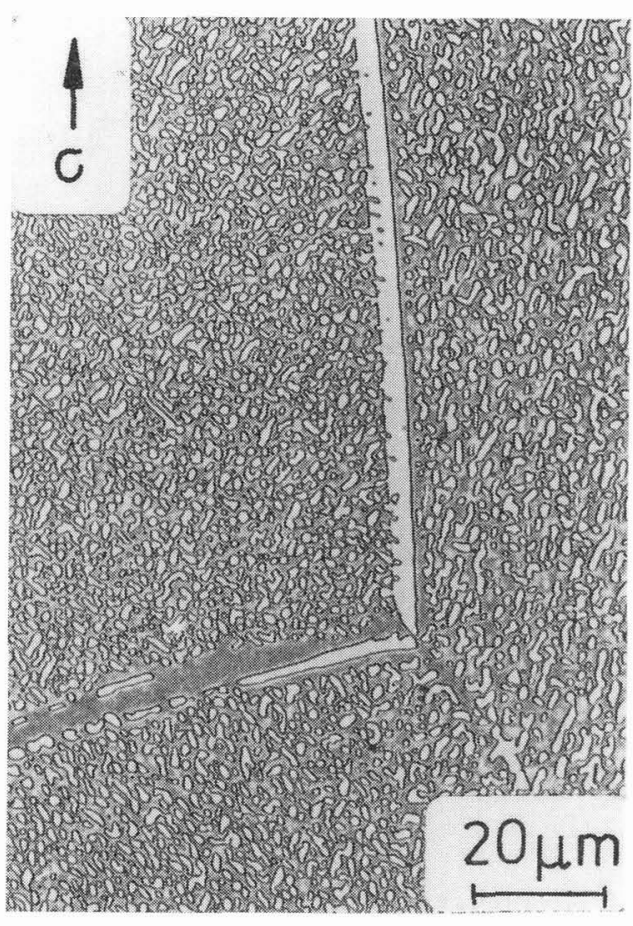

Figure 5: Optical micrograph of a $\mathrm{Ni}-\mathrm{Al}-\mathrm{Fe}$ alloy with 20 vol.\% intermetallic $\mathrm{NiAl}$ precipitate particles after tensile creep at $800^{\circ} \mathrm{C}$ for 2 years with $1 \%$ elongation (the direction of applied stress $\sigma$ is indicated by an arrow) [14].
Finally it is noted that alloys with oriented precipitate particles may be attractive for applications because of anisotropic physical and mechanical properties. However, particle orienting by selective coarsening is a time consuming process which is disadvantageous with respect to processing efficiency.

The discussed cases of particle orienting can be regarded as simple model cases for the effect of external stresses on two particulate co-existing precipitate phases in a common matrix. Then the particles of the different phases may interact with the external stress to produce different interaction energies which may enlarge the usually small solubility differences between the two precipitate phases to such an extent that the favoured phase coarsens at the expense of the other one which then dissolves [1].

Besides the discussed direct effects of external stresses on the particle behaviour, the matrix behaviour is affected by the presence of external elastic stresses, too as becomes visible during diffusion creep. The application of an elastic tensile stress, which is not sufficient for the generation and movement of dislocations, to an alloy results in stress-directed diffusional flow of atoms from surfaces and interfaces parallel to the stress to those perpendicular to the stress which leads to elongation and is known as diffusion creep [15-18]. In the case of two-phase alloys with precipitate particles the diffusional flow of the matrix leads to particle coagulation and grain boundary formation at the grain boundaries parallel to the applied stress and formation of a precipitatefree matrix layer at the grain boundaries perpendicular to the applied stress since the particles at the former grain boundaries are still in local equilibrium with the matrix and the "new" matrix at the latter boundaries lacks supersaturation for re-precipitation of particles. This process of matrix re-distribution is illustrated by Fig. 5 . It is noted that the precipitate-free zone grows in thickness with increasing elongation, and the ratio of zone thickness and grain length is indeed about $1 \%$ in the case of Fig. 5, which corresponds to the measured elongation of the specimen.

\section{EFFECTS OF PLASTIC STRAIN}

\subsection{Nucleation}

External elastic stresses that are higher than the yield stress produce plastic deformation of the material with generation and motion of dislocations which are linear defects with an excess line energy and a surrounding cylindrical elastic stress field. Nucleation on dislocations may be energetically advantageous for coherent particles with misfit, if the particle misfit is accommodated by the elastic strain of the dislocation stress field, i.e. if there is an attractive interaction between the dislocation stress field and the particle stress field. Nucleation on dislocations may also be energetically advantageous for incoherent particles since the dislocation line is then interrupted by the particle which shortens the dislocation and saves dislocation energy [1]. In any case the number of available dislocations has to be suf- 
ficiently large compared with the number of nuclei for achieving a significant effect on nucleation, i.e. the deformation rate has to be sufficiently high compared with the nucleation rate as was estimated previously [19].
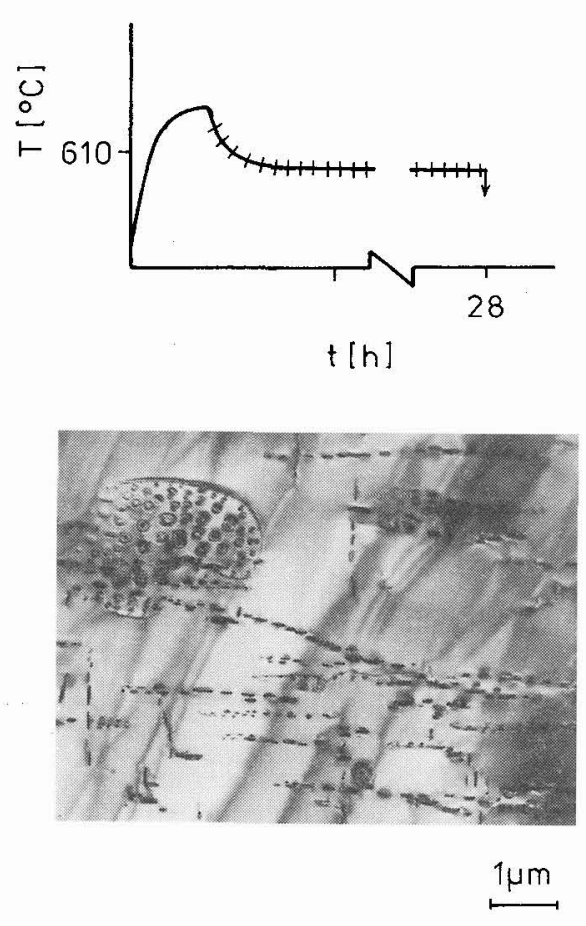

Figure 6: Transmission electron micrograph of precipitated Au particles in a Fe-Mo-Au single crystal with applied external elastic tensile stress of $25 \mathrm{MPa}$ during nucleation and coarsening; the temperature vs. time diagram indicates the used heat treatment $\lceil 20\rceil$.
Apart from the rôle of dislocations as favourable nucleation sites, dislocations may act as sinks for the segregation of solute atoms. This reduces the matrix supersaturation and thereby the nucleation rate $[19,21]$.

Preferential nucleation on dislocations is exemplified by the already discussed case of $\alpha$ " $\mathrm{Fe}_{10} \mathrm{~N}_{2}$ precipitation in $\mathrm{Fe}-\mathrm{N}$ alloys where nucleation is enhanced dramatically by prior cold-rolling [22] - see [1]. Another example is the case of the precipitation of Au particles with particle colony formation in $\mathrm{Fe}-\mathrm{Mo}$-Au alloys where homogeneous nucleation is observed only for sufficient initial undercooling whereas nucleation occurs heterogeneously at dislocations for insufficient initial undercooling [20] as is visible in Fig. 6 . In this case the attractive interaction between nucleated particle and dislocation obviously diminishes during growth with transition to incoherency to such an extent that the dislocation detaches itself from the growing particle and migrates into the still supersaturated matrix with further nucleation of particles. The driving force for this process results from both the supersaturation and the elastic forces of the dislocation.

The effect of dislocations on nucleation is used for controlling the precipitation process - and related microstructure reactions - by prior or simultaneous plastic deformation which is known as thermomechanical processing $[1,23,24]$. The plastic strain with generation, multiplication and motion of dislocations initiates nucleation, and the resulting particle distribution determines and stabilizes the grain size distribution by controlling the crystallization process. Both the particle and grain size distributions sensitively control the mechanical behaviour of the material as characterized by strength and toughness, and thus thermomechanical processing has found wide application for producing structural alloys with optimized mechanical properties. The most eminent example are the microalloyed steels which combine high strength and high toughness and are also known as HSLA or highstrength low-alloy steels $[25,26]$. The mutual interaction of deformation and precipitation with strain induced nucleation was analysed and modelled in detail [27].

\subsection{Growth and Coarsening}

Dislocations as well as grain boundaries are paths of easy diffusion which enhance the exchange of material between coarsening particles. Indeed the kinetics of Ostwald ripening is affected if the coarsening particles are interconnected by dislocations or grain boundaries, and respective theoretical models are available $[21,28]$. However, during plastic straining dislocations move and attach and detach themselves to and from particles, i.e. particles are only partially connected by dislocations. Thus a 
significant can only be expected when the dislocation density $\rho$ is sufficiently high, i.e. when the dislocation distance $\rho^{-1 / 2}$ is sufficiently small compared with the particle distance [19,21].

During deformation - in particular at high-temperatures - dislocations move not only conservatively by dislocation glide, but also nonconservatively by dislocation climb with production of excess vacancies which accelerates bulk diffusion and thereby Ostwald ripening. Again the deformation rate has to be sufficiently high for producing a significant effect since the excess vacancies heal out by normal recovery[19,21].

Coherent growing particles may attract dislocations which relaxes the coherency stress. This effect contributes to the already mentioned transition to incoherency, i.e. the coherent-to-incoherent transition is accelerated $[19,21]$ which affects the kinetics of coarsening as discussed in Section 3.2. In addition, a dislocation may move through the particle by which the particle is sheared and finally cut into parts if a sufficient number of dislocations shear the particle on the same glide plane. This process of particle shearing is counteracted by short-range diffusion in the particle and at the surface to restore the original particle shape with minimum interface energy, i.e. particle shearing is observed only for sufficiently high deformation rates. This process of destabilization and subdivision of precipitates is analogous to the spheroidization of pearlite in steels which is accelerated dramatically by concurrent fast deformation [29-32].

It is concluded that the precipitation process is affected in a significant way by concurrent deformation only if the deformation rate is sufficiently high compared with the precipitation rate, and simple estimates were presented previously [19,21]. Thus effects can be expected for fast deformation - e.g. rolling - as is used for materials processing whereas negligible effects are expected in general for slow deformation during materials service, in particular creep. Special situations with effects even by creep deformation may occur if there is Ostwald ripening of several competing phases. Such effects were discussed with respect to the steel $10 \mathrm{CrMo} 910\left(2 \frac{114}{4} \mathrm{Cr}, 1 \% \mathrm{Mo}\right)$ where various carbide phases precipitate simultaneously and sequentially with inhomogeneous particle distributions in the grains and on grain boundaries [33]. It was found that - apart from direct effects of creep on the microstructure, e.g. changes in grain size distribution and/or formation of precipitate-free zones - the effects of creep on growth and coarsening are still negligibly small under practical service conditions with long-term creep with rates of $10^{-10} 1 / \mathrm{s}$ or less.

\section{CONCLUSIONS}

The various stages of precipitation by nucleation, growth and coarsening my be influenced by concurrent elastic and/or plastic deformation depending on the ratio of the driving forces for precipitation and deformation or on the ratio of the rates of precipitation and deformation. Various effects can be produced with respect to precipitation kinetics and distribution of precipitated particles by appropriate choice of boundary conditions of precipitation and deformation. The physical and mechanical properties of a material depend in a sensitive way on the material microstructure and in particular on the distribution of precipitated particles. Thus the discussed effects are of practical importance for controlling and optimizing the materials properties, and indeed thermomechanical processing has found widespread application for materials production. For this fast processing is most attractive, and thus deformation effects on nucleation are of primary importance whereas changes in precipitate distribution by growth and coarsening are time consuming which usually precludes application.

\section{References}

[1] Pitsch W. and Sauthoff G. , "Microstructure of Steels", Steel, Vol. 1, Verein Deutscher Eisenhüttenleute Ed. (Springer Verlag, Berlin, 1992) pp. 1-166.

[2] Denbigh K. , The Principles of Chemical Equilibrium (University Press, Cambridge, 1971) pp.1494. 
[3] Binder K. , "Spinodal Decomposition", Materials Science and Technology - Vol. 5: Phase Transformations in Materials, R.W. Cahn, P. Haasen, and E.J. Kramer Eds. (VCH, Weinheim, 1991) pp. 405471.

[4] Sauthoff G. , Z. Metallkde. 66 (1975) 106-109.

[5] Sauthoff G. , Z. Metallkde. 67 (1976) 25-29.

[6] Cohen J.B. , Metall. Mat. Trans. A 25 (1994) 2565-2568.

[7] Tanaka Y., Sato A. and Mori T. , Acta Metall. 26 (1978) 529-540.

[8] Sauthoff G. , Acta Metall. 29 (1981) 637-642.

[9] Hort W. and Johnson W.C., Metall. Mat. Trans. A 25 (1994) 2695-2703.

[10] Sauthoff G. , Z. Metallkde. 68 (1977) 500-505.

[11] Neuhäuser H.J. and Pitsch W. , Z. Metallkde. 62 (1971) 792-796.

[12] Sauthoff G. , Z. Metallkde. 68 (1977) 22-26.

[13] Sauthoff G. and Pitsch W., Phil. Mag. B 56 (1987) 471-483.

[14] Jung I. and Sauthoff G. , "The Effect of Ordered Precipitates on Creep in Ferritic Fe-Ni-Al Alloys", Creep and Fracture of Engineering Materials and Structures, B. Wilshire and R.W. Evans Eds. (The Institute of Metals, London, 1987) pp. 257-270.

[15] Herring C. , J. Appl. Phys. 21 (1950) 437-445.

[16] Coble R.L. , J. Appl. Phys. 34 (1963) 1679-1683.

[17] Greenwood G.W., "Grain Boundaries as Vacancy Sources and Sinks", Vacancies 76, R.E. Smallman and J.E. Harris Eds. (The Metals Society, London, 1977) pp. 141-148.

[18] Greenwood G.W., Scripta Metall. Mater. 30 (1994) 1527-1530.

[19] Sauthoff G. , Arch. Eisenhüttenwes. 54 (1983) 155-159.

[20] Sauthoff G. , phys. stat. sol. (a) 26 (1974) K5-K7.

[21] Sauthoff G. , "Influence of Service Conditions on Phase Development and Transformation", Phase Stability in High Temperature Alloys, V. Guttmann Ed. (Appl. Science Publ. Ltd. Barking, 1981) pp. 93-118.

[22] Pitsch W. , "Elektronenmikroskopische Untersuchungen der Eisennitridausscheidungen im Ferrit", Stickstoff in Metallen, Akademie-Verlag, Berlin, 1965) pp. 118-128.

[23] Bleck W., Müschenborn W. and Meyer L., steel research 59 (1988) 344-351.

[24] Dahl W., Hagen M., Karhausen K., Kaspar R. and Meyer L., Stahl u. Eisen 111 (1991) 113-119.

[25] Bleck W., Meyer L. and Kaspar R. , Stahl u. Eisen 111 (1991) 51-56.

[26] Biegus C., Evertz T. and Dahl W. , Stahl u. Eisen 114 (1994) 65-70.

[27] Dutta B., Valdes E. and Sellars C.M. , Acta Metall. Mater. 40 (1992) 653-662.

[28] Hoyt J.J. , Acta Metall. Mater. 39 (1991) 2091-2098.

[29] Gautier-Aeby E. , Transformation perlitique sous contrainte de traction d'un acier eutectoide étude cinetique et comportement mécanique (Dr.-Ing. Thèse, Institut National Polytechnique de Lorraine (Nancy), 1978).

[30] Nijhof G.H. , Accelerated Spheroidization of Cold Rolled Eutectoid Steel (Dr.-Ing. Proefschrift, Technische Hogeschool Delft, 1981).

[31] Chattopadhyay S. and Sellars C.M. , Acta Metall. 30 (1982) 157-170.

[32] Kaspar R., Kapellner W. and Lang C. , steel research 59 (1988) 492-497.

[33] Schulze O. and Sauthoff G. , Arch. Eisenhïttenwes. 55 (1984) 73-80. 